\title{
Miniexame do Estado Mental: evidências de validade baseadas na estrutura interna
}

\author{
Denise Mendonça de Meloํㅜ Altemir José Gonçalves Barbosa \\ Universidade Federal de Juiz de Fora, Juiz de Fora-MG, Brasil \\ Anita Liberalesso Neri \\ Universidade Estadual de Campinas, Campinas-SP, Brasil
}

\section{RESUMO}

Em razão da escassez de pesquisas que investigaram empiricamente a dimensionalidade do Miniexame do Estado Mental (MEEM), este estudo teve como objetivo obter evidências de validade com base na estrutura interna para essa medida por meio de análise de componentes principais (ACP) e de análise fatorial confirmatória (AFC). A amostra de 2.734 idosos da comunidade foi dividida aleatoriamente em dois grupos: ACP $(n=1.361)$ e AFC $(n=1.373)$. Na ACP, uma solução com cinco componentes - Habilidades Escolares, Orientação, Memória de Curto Prazo, Memória - Evocação e Memória - Reconhecimento — foi a mais adequada. Quatro modelos de estrutura interna foram testados com AFC, incluindo o formado na ACP. Três modelos com estrutura multidimensional de cinco fatores e um fator de segunda ordem apresentaram índices de ajuste satisfatórios, o que não ocorreu com o modelo unidimensional. Assim, o MEEM parece ser uma medida multidimensional, mas estudos adicionais são recomendados.

Palavras-chave: envelhecimento; avaliação neuropsicológica; análise fatorial.

\section{ABSTRACT - Minimental State Examination: Validity evidence based on internal structure}

Studies that empirically investigate the dimensionality of the Minimental State Examination (MMSE) are few. For this reason, this study aimed to obtain validity evidence based on internal structure for this measure by principal component analysis (PCA) and confirmatory factor analysis (CFA). The sample of 2,734 community-dwelling elderly people was randomly divided into two groups: PCA $(n=1,361)$ and CFA $(n=1,373)$. In the PCA, a five components solution was the best — School Skills, Guidance, Short Term Memory, Memory Evocation and Memory - Recognition. Four internal structure models were tested with CFA, including the PCA formed. Three models with a multidimensional structure of five factors and a factor of second order showed satisfactory fit indices, which did not happen with the one-dimensional model. Therefore, the MMSE seems to be a multidimensional measure, but further studies are recommended.

Keywords: aging; neuropsychological assessment; factor analysis.

RESUMEN - Mini Examen del Estado Mental: evidencia de validez basada en la estructura interna

Debido a la escasez de los trabajos que investigan empíricamente la dimensionalidad del Mini Examen del Estado Mental (MEEM), este estudio tiene como objetivo obtener evidencia de validez basada en la estructura interna de esta medida por análisis de componentes principales (PCA) y el análisis factorial confirmatorio (AFC). La muestra de 2.734 adultos mayores residentes en la comunidad se dividió aleatoriamente en dos grupos: ACP $(n=1.361)$ y AFC $(n=1.373)$. En el ACP, una solución con cinco componentes - Competencias en la Escuela, Dirección, Memoria a Corto plazo, Memoria - Evocación y Memoria de Reconocimiento — fue la más apropiada. Cuatro modelos de estructuras internas fueron probados con AFC, incluyendo el formado en la ACP formado. Tres modelos con estructura multidimensional de cinco factores y un factor de segundo orden mostraron índices de ajuste satisfactorios, lo que no ocurrió con el modelo unidimensional. Así, el MEEM parece ser una prueba multidimensional, sin embargo se recomiendan más estudios.

Palabras clave: envejecimiento; evaluación neuropsicológica; análisis factorial.

O Miniexame do Estado Mental (MEEM) (Folstein, Folstein, \& McHugh, 1975) é uma medida dicotômica de 30 itens, com aplicação rápida e breve, que rastreia o comprometimento das funções cognitivas. Avalia orientação temporal e espacial, memória imediata e de evocação de palavras, cálculo, nomeação, repetição, execução de um comando, leitura, escrita e habilidade visomotora (Bertolucci, Brucki, Campacci, \& Juliano, 1994; Brucki,
Nitrini, Caramelli, Bertolucci, \& Okamoto, 2003; Mitolo et al., 2014).

Os itens do MEEM foram categorizados por Folstein et al. (1975) em cinco dimensões baseadas em análise teórica e prática clínica: Orientação $\left(1^{\circ}\right.$ ao $10^{\circ}$ itens), Registro (11 $1^{\circ}$ ao $13^{\circ}$ itens), Atenção e Cálculo (14 ${ }^{\circ}$ ao $18^{\circ}$ itens), Recordação $\left(19^{\circ}\right.$ ao $21^{\circ}$ itens) e Linguagem $\left(22^{\circ}\right.$ ao $30^{\circ}$ itens). Todavia, essa divisão pode 
não representar a estrutura interna empírica da medida (Tinklenberg et al., 1990), uma vez que não tem sido rigorosamente estudada (Jones \& Gallo, 2000). Do ponto de vista teórico, a classificação efetuada por Folstein et al. (1975) apresenta problemas, uma vez que, por exemplo, falta precisão terminológica no caso de Atenção e Cálculo. Nos itens que compõem essa dimensão é solicitado que o avaliado efetue subtrações, mas não é claro se o nível de atenção demandado pela tarefa é diferente daquele necessário nos demais itens do instrumento.

O primeiro estudo empírico sobre a dimensionalidade do MEEM foi, provavelmente, o realizado por Fillenbaum, Heyman, Wilkinson, e Haynes (1987). Desde então, pesquisas internacionais (Abraham et al., 1994; Baños \& Franklin, 2002; Braekhus, Laake, \& Engedal, 1992; Brugnolo et al., 2009; Commenges et al., 1992; Ideno, Takayama, Hayashi, Takagi, \& Sugai, 2012; Jones \& Gallo, 2000; Tinklenberg et al., 1990) e nacionais (CastroCosta et al., 2009, 2014) têm investigado a estrutura interna do exame por meio de análises fatoriais exploratórias e/ou confirmatórias e de componentes principais.

As pesquisas de Castro-Costa et al. (2009; 2014) merecem uma atenção especial por serem os únicos estudos nacionais dentre os supracitados e por terem utilizado amostras comunitárias e analisado a estrutura interna da versão de 30 itens do instrumento. Os autores identificaram que o MEEM - versão de Seabra, Concilio, Villares, e Carlini (1990) - é de natureza multidimensional e formado por cinco componentes ou fatores: Concentração ( $14^{\circ}$ ao $18^{\circ}$ itens), Linguagem e Praxia $\left(5^{\circ}, 24^{\circ}, 25^{\circ}\right.$ e $28^{\circ}$ ao $30^{\circ}$ itens), Orientação ( $1^{\circ}$ ao $4^{\circ}, 6^{\circ}$ ao $10^{\circ}, 26^{\circ}$ e $27^{\circ}$ itens), Atenção $\left(11^{\circ}\right.$ ao $13^{\circ}$ itens $)$ e Memória $\left(19^{\circ}\right.$ ao $23^{\circ}$ itens). Essa estrutura interna foi obtida a partir de uma análise de componentes principais (ACP) e análises fatoriais confirmatórias (AFC). É preciso salientar que a ACP revela um modelo formativo, ou seja, itens causam determinado componente, não sendo, portanto, um modelo de traços latentes, como as análises fatoriais, pois, nesse caso, o fator causa a variabilidade dos itens (Beavers et al., 2013; Markus \& Borsboom, 2011).

A amplitude da avaliação realizada pelo MEEM e sua praticidade gerou grande aceitação pelas comunidades científica e clínica (Molloy \& Standish, 1997). Muitas traduções para diversos idiomas (Melo \& Barbosa, 2015) e versões diferentes no mesmo idioma (Folstein, Folstein, \& McHugh, 2007) são encontradas. Atualmente, é um instrumento de rastreio de declínio cognitivo amplamente utilizado (Kim et al., 2014). Entretanto, pesquisadores brasileiros (Vasconcelos, Brucki, \& Bueno, 2007; Melo \& Barbosa, 2015) e de outros países (Beyermann et al., 2013; Hoops et al., 2009; Spencer et al., 2013) têm questionado suas propriedades psicométricas. É preciso salientar que instrumentos que medem um construto psicológico, como o MEEM, precisam preencher requisitos mínimos, entre eles, apresentar evidências de validade, fidedignidade ou confiabilidade e padronização (AERA, APA, \& NCME, 2004).
Este estudo teve como objetivo analisar a estrutura interna do MEEM por meio de ACP e AFC. No último caso, foram contrastados os componentes obtidos com a ACP, as dimensões teóricas originais do instrumento (Folstein et al., 1975) e os componentes/fatores do único estudo brasileiro encontrado sobre a dimensionalidade do exame (Castro-Costa et al., 2009).

\section{Método}

\section{Participantes}

Este artigo foi realizado com dados do estudo multicêntrico e multidisciplinar "Fragilidade em Idosos Brasileiros (FIBRA)" do polo coordenado pela Universidade Estadual de Campinas (Unicamp). Critérios de exclusão e inclusão dos participantes e demais procedimentos de amostragem do estudo FIBRA, polo Unicamp, são apresentados em Neri et al. (2013). Salienta-se que o estudo FIBRA foi aprovado pelo Comitê de Ética em Pesquisa com Seres Humanos da Faculdade de Ciências Médicas da Unicamp (parecer 208/2007).

Foram utilizados dados de 2.734 idosos com 65 anos ou mais, que, de acordo com os objetivos e a análise dos dados (ver Procedimento), foram divididos aleatoriamente em dois subgrupos - ACP $(n=1.361)$ e AFC $(n=1.373)$ - , cada um composto por aproximadamente $50 \%$ dos participantes (Tabela 1). A idade média em anos dos participantes foi $72,72(D P=5,88)$, e a média dos anos de escolaridade foi $4,57(D P=4,01)$.

\section{Instrumentos}

O estudo FIBRA utilizou a versão brasileira do MEEM adaptada por Brucki et al., 2003, com notas de corte derivadas desse estudo, que são recomendadas pelo Departamento Científico de Neurologia Cognitiva e do Envelhecimento da Academia Brasileira de Neurologia para rastreio de declínio cognitivo sugestivo de demência (Nitrini et al., 2005), menos um desvio padrão. Dessa forma, os escores de corte adotados foram 17 para idosos analfabetos, 22 para idosos com escolaridade entre um e quatro anos, 24 para idosos com escolaridade entre cinco e oito anos, e 26 para idosos com mais de nove anos de escolaridade. Para caracterizar a amostra em termos demográficos, foram utilizados dados sobre idade, sexo e escolaridade, encontrados no banco eletrônico do Estudo FIBRA.

\section{Procedimento}

O processo de recrutamento e a coleta de dados do Estudo FIBRA também são descritos detalhadamente em Neri et al. (2013). Não obstante, é preciso esclarecer que, em síntese, foram realizadas visitas domiciliares para recrutar os participantes, que, se satisfizessem os critérios de inclusão, eram convidados a se deslocar em data e horários previamente agendados para um local de coleta de dados. Tanto o MEEM quanto o questionário demográfico constituíram parte de uma sessão única de coleta e foram 
aplicados na forma de entrevista, e, em média, o tempo de resposta dos dois instrumentos foi de 15 minutos.

Para análise de dados, provas de estatística descritiva (média e desvio padrão) e inferencial (teste $t$ de Student) foram utilizadas. Adotou-se para todos os testes um nível de significância de $5 \%$.

Repetiu-se parcialmente a busca de evidências de validade do MEEM baseadas na estrutura interna realizada por Castro-Costa et al. (2009) ainda que a ACP por eles utilizada não seja um modelo de variáveis latentes (Beavers et al., 2013; Markus \& Borsboom, 2011). Os testes de Kaiser-Meyer-Olkin (KMO) e de Esfericidade de Bartlett foram adotados para avaliar a adequação dos dados para a análise fatorial. Adotouse, também, o método de rotação oblíqua de fatores Promax. Para efetuar essa análise, foi utilizada a versão 20 do software estatístico SPSS.

Para definição do número dos componentes que seriam extraídos, optou-se, porém, por utilizar as análises paralelas e não o teste do screeplot empregado pelos autores. A literatura (Damásio, 2012) tem destacado que a escolha dos fatores a serem retidos com base na inspeção visual de um gráfico de sedimentação é subjetiva e muitas vezes ambígua por ser obtida por um processo visual e, desse modo, desaconselha seu uso.

As cargas fatoriais da ACP foram consideradas significativas quando excederam o valor 0,30 . Elas foram avaliadas como excelentes quando maiores do que 0,71 , muito boas quando variaram de 0,63 a 0,70 , boas quando entre 0,55 a 0,62 , razoáveis quando entre 0,45 e 0,53 e pobres quando entre 0,32 e 0,44 (Laros, 2012).

AFCs testaram quatro modelos de estrutura interna do MEEM:

1. M1 - modelo unidimensional;

2. M2 - modelo baseado na ACP deste estudo com cinco fatores e um fator de segunda ordem;

3. M3 - modelo baseado em Folstein et al. (1975) com cinco fatores e um fator de segunda ordem; e

4. $\quad$ M4 - modelo de Castro-Costa et al. (2009; 2014) com cinco fatores e um fator de segunda ordem.

Tabela 1

Caracterização demográfica das amostras

\begin{tabular}{|c|c|c|c|c|c|c|}
\hline \multirow{3}{*}{ Variáveis } & \multicolumn{4}{|c|}{ Subgrupos } & \multirow{2}{*}{\multicolumn{2}{|c|}{ Total }} \\
\hline & \multicolumn{2}{|c|}{ AFC } & \multicolumn{2}{|c|}{ ACP } & & \\
\hline & $n$ & $\%$ & $n$ & $\%$ & $n$ & $\%$ \\
\hline \multicolumn{7}{|l|}{ Localidades $^{\mathrm{a}}$} \\
\hline Campinas - SP & 410 & 29,86 & 409 & 30,05 & 819 & 29,96 \\
\hline Belém - PA & 316 & 23,02 & 351 & 25,79 & 667 & 24,40 \\
\hline Ermelino Matarazzo - São Paulo - SP & 181 & 13,18 & 161 & 11,83 & 342 & 12,51 \\
\hline Poços de Caldas - MG & 153 & 11,14 & 124 & 9,11 & 277 & 10,13 \\
\hline Parnaíba - PI & 131 & 9,54 & 143 & 10,51 & 274 & 10,02 \\
\hline Campina Grande - PB & 90 & 6,55 & 91 & 6,69 & 181 & 6,62 \\
\hline Ivoti - RS & 92 & 6,70 & 82 & 6,02 & 174 & 6,36 \\
\hline \multicolumn{7}{|l|}{$\mathrm{Sexo}^{\mathrm{a}}$} \\
\hline Feminino & 923 & 67,23 & 903 & 66,35 & 1826 & 66,79 \\
\hline Masculino & 450 & 32,77 & 458 & 33,65 & 908 & 33,21 \\
\hline \multicolumn{7}{|l|}{ Idade (anos) $)^{a}$} \\
\hline $65-69$ & 490 & 35,69 & 492 & 36,15 & 982 & 35,92 \\
\hline $70-74$ & 408 & 29,72 & 434 & 31,89 & 842 & 30,80 \\
\hline $75-79$ & 277 & 20,17 & 251 & 18,44 & 528 & 19,31 \\
\hline 80 ou mais & 198 & 14,42 & 184 & 13,52 & 382 & 13,97 \\
\hline \multicolumn{7}{|l|}{ Escolaridade (anos de estudo) b } \\
\hline Analfabeto ou não escolarizado & 232 & 16,90 & 212 & 15,59 & 444 & 16,25 \\
\hline $1-4$ & 718 & 52,29 & 656 & 48,24 & 1374 & 50,27 \\
\hline $5-8$ & 254 & 18,50 & 266 & 19,56 & 520 & 19,03 \\
\hline 9 ou mais & 169 & 12,31 & 226 & 16,62 & 395 & 14,45 \\
\hline
\end{tabular}

Nota: ${ }^{a} n=2.734$. ${ }^{b} n=2.733$; um participante não informou; $\mathrm{AFC}=$ análise fatorial confirmatória; $\mathrm{ACP}=$ análise de componentes principais. 
As análises foram realizadas no software MPlus (versão 7.11), utilizando o método de estimação Weighted Least Squares Mean and Variance Adjusted (WLSMV), adequado para natureza dicotômica das variáveis (Muthén \& Muthén, 2012). Para analisar o ajustamento dos modelos, foram utilizados os indicadores e os valores de referência propostos por Marôco (2010): razão do qui-quadrado pelos graus de liberdade ( $\left.\chi^{2} / g .1.\right)$; Comparative Fit Index (CFI) e Tucker-Lewis Index (TLI); Root Mean Square Error of Aproximation (RMSEA); e Expected Cross-validation Index (ECVI). Visto que o software MPlus não gera o ECVI, este indicador foi calculado com a fórmula $\left(\chi^{2} / \mathrm{N}-1\right)+(\mathrm{a} / \mathrm{N}-1)$, em que "a" significa o número de parâmetros do modelo.

\section{Resultados}

A pontuação média no MEEM foi 23,96 $(D P=4,10)$. Como esperado por terem sido compostas aleatoriamente, não foram obtidas diferenças significativas $\left(t_{o}(2734\right.$; $2732)=0,985 ; p=0,324)$ entre os escores das amostras da ACP $(M=24,03 ; D P=4,23)$ e da AFC $(M=23,88$; $D P=3,97)$. Quanto à sugestão de declínio cognitivo, a prevalência foi de $21,33 \%(n=583)$ para a amostra total.

Como os resultados dos testes de $\operatorname{KMO}(0,880)$ e Esfericidade de Bartlett $\left(\chi^{2}(6818,884)=435 ; p=0,000\right)$ indicaram que os dados eram adequados para a ACP, procedeu-se à análise paralela, que revelou que cinco componentes deveriam ser retidos. Assim, realizou-se a análise programando o software para reter cinco componentes (Tabela 2). Juntos eles explicam 37,632\% da variância das respostas e apresentaram autovalores entre 1,245 e 5,344.

O primeiro componente foi denominado Habilidades Escolares por englobar cálculo, leitura, redação e reprodução visomotora com uma ferramenta essencialmente escolar (caneta) e, mais especificamente, por incluir os cinco itens do sete seriado, sendo quatro deles com excelentes cargas fatoriais e um deles (100 menos 7) com carga muito boa. Os itens de escrita da frase e de leitura e execução da tarefa de fechar os olhos tiveram cargas razoáveis, e o item de cópia do desenho dos pentágonos carga pobre. Orientação foi a designação eleita para o segundo componente, pois os dez itens de orientação espacial e temporal apresentaram cargas fatoriais mais elevadas nele, sendo um deles (local mais amplo) com carga muito boa, três deles (mês, local restrito e dia) com cargas entre razoáveis e boas, quatro itens (dia da semana, estado, bairro e ano) com cargas razoáveis e dois (hora e cidade), com cargas pobres. O terceiro componente, Memória de Curto Prazo, incluiu os três itens de recordação imediata (tijolo, vaso, carro) com cargas, respectivamente, excelente, muito boa e boa, e a repetição da frase (nem aqui, nem ali, nem lá) apresentou carga boa, além de dois itens do comando de três estágios, um deles com carga pobre (colocar o papel no chão) e o outro com carga não significativa (dobrar o papel ao meio). O quarto componente foi nomeado como Memória - Evocação porque incluiu os três itens de recordação tardia (carro, tijolo, vaso) que obtiveram cargas muito boas. O quinto componente, Memória - Reconhecimento, contém os dois itens de identificação de objetos (relógio e caneta) com cargas muito boas e o primeiro item do comando de três estágios (pega a folha) com carga pobre.

As AFCs (Tabela 3) revelaram que o M2 - modelo derivado da ACP apresentada neste estudo - assim como o modelo original de Folstein et al. (1975) (M3), é estatisticamente melhor que os demais que foram testados. Com base no ECVI, o M2 foi o melhor modelo. Tanto o M1 quanto o M4 apresentaram pelo menos um índice de ajuste insatisfatório.

\section{Discussão}

Ao buscar evidências de validade baseadas na estrutura interna para o MEEM, constatou-se que se trata de uma medida multidimensional, convergindo com Schultz-Larsen, Kreiner e Lomholt (2007) e Brugnolo et al. (2009). Tanto a ACP quanto a AFC revelaram que o MEEM contém cinco componentes ou fatores. No último caso, dois modelos fatoriais analisados se mostraram estatisticamente aceitáveis - M2 e M3. Além disso, parece que os componentes identificados com ACP nesta pesquisa também podem ser considerados fatores, pois representaram o melhor modelo na AFC, sendo, desse modo, os mais adequados para explicar a estrutura interna dessa medida de funções cognitivas, que pode resultar em sugestão de declínio cognitivo em idosos.

Do ponto de vista estatístico, há que se esclarecer que são numerosas as pesquisas que buscam determinar se a ACP e as análises fatoriais geram soluções diferentes (Beavers et al., 2013). Isso não ocorreu no estudo aqui descrito, convergindo com relatos (ver, por exemplo, Beavers et al., 2013) de que ambos os processos podem gerar soluções semelhantes.

A análise da estrutura interna de um teste pode indicar o quanto as relações entre os itens do teste e as suas dimensões estão em conformidade com o construto teórico que ancora um teste e, consequentemente, com as propostas de interpretações de seus escores (AERA et al., 2004). Acrescenta-se que a estrutura de um teste pode implicar em uma única dimensão do comportamento ou pode postular várias, como parece ser o caso do MEEM.

Dentre os cinco componentes do MEEM identificados pela ACP deste estudo, o primeiro destacou-se por agrupar oito itens - que envolvem cálculo, leitura e reprodução visomotora com caneta -, os quais exigem aptidões em tarefas típicas do contexto escolar. Por isso, foi chamado de Habilidades Escolares. Muitas pesquisas têm destacado que a escolaridade influencia o desempenho no MEEM (Bertolucci et al., 1994; Brucki, Mansur, Carthery-Goulart, \& Nitrini, 2011) e, consequentemente, deve ser considerada ao se estabelecerem pontos de corte. Alerta-se que não somente a quantidade de anos estudados é importante mas também a qualidade do ensino recebido e sua influência sobre o desenvolvimento cognitivo, especialmente na velhice (Aprahamian, Martinelli, Cecato, \& Yassuda, 2011) e em países em desenvolvimento, como o Brasil, onde a educação formal é muito heterogênea (Farfel et al., 2013). 
Tabela 2

Análise de Componentes Principais do Miniexame do Estado Mental

\begin{tabular}{|c|c|c|c|c|c|}
\hline \multirow[b]{2}{*}{ Itens do MEEM } & \multicolumn{5}{|c|}{ Componentes } \\
\hline & $\begin{array}{l}\text { Habilidades } \\
\text { Escolares }^{a}\end{array}$ & Orientação ${ }^{b}$ & $\begin{array}{l}\text { Memória de } \\
\text { Curto Prazo }\end{array}$ & $\begin{array}{l}\text { Memória - } \\
\text { Evocaçãod }^{\mathrm{d}}\end{array}$ & $\begin{array}{c}\text { Memória - } \\
\text { Reconhecimento }\end{array}$ \\
\hline 17. Quanto é 79 - 7 & 0,813 & & & & \\
\hline 18. Quanto é $72-7$ & 0,803 & & & & \\
\hline 16. Quanto é $86-7$ & 0,785 & & & & \\
\hline 15. Quanto é 93 - 7 & 0,752 & & & & \\
\hline 14. Quanto é 100 - 7 & 0,654 & & & & \\
\hline 29. Escrever uma frase & 0,467 & & & & \\
\hline 28. Feche os olhos & 0,439 & & & & \\
\hline 30. Cópia dos pentágonos & 0,360 & & & & \\
\hline 6. Que local é este aqui? & & 0,681 & & & \\
\hline 2. Em que mês estamos? & & 0,535 & & & \\
\hline 7. Em que local nós estamos? & & 0,511 & & & \\
\hline 1. Que dia é hoje? & & 0,503 & & & \\
\hline 4. Em que dia da semana? & & 0,479 & & & \\
\hline 10. Em que estado nós estamos? & & 0,478 & & & \\
\hline $\begin{array}{l}\text { 8. Em que bairro nós estamos ou } \\
\text { qual o nome de uma rua próxima? }\end{array}$ & & 0,451 & & & \\
\hline 3. Em que ano? & & 0,416 & & & \\
\hline 5. Que horas são agora? & & 0,336 & & & \\
\hline 9. Em que cidade nós estamos? & & 0,320 & & & \\
\hline 13. Tijolo & & & 0,745 & & \\
\hline 12. Vaso & & & 0,627 & & \\
\hline 11. Carro & & & 0,555 & & \\
\hline 24. Nem aqui, nem ali, nem lá & & & 0,544 & & \\
\hline 27. Coloca no chão & & & 0,347 & & \\
\hline 26. Dobra ao meio & & & 0,261 & & \\
\hline 19. Carro & & & & 0,676 & \\
\hline 21. Tijolo & & & & 0,660 & \\
\hline 20. Vaso & & & & 0,626 & \\
\hline 22. Relógio & & & & & 0,684 \\
\hline 23. Caneta & & & & & 0,653 \\
\hline 25. Pega a folha com a mão direita & & & & & 0,313 \\
\hline
\end{tabular}

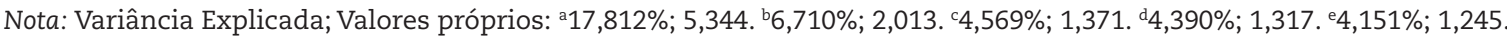
MEEM=Miniexame do Estado Mental.

Tabela 3

Análise Fatorial Confirmatória do Miniexame do Estado Mental

\begin{tabular}{lccccc}
\hline Modelos fatoriais & $\chi^{2} / g l^{\mathrm{a}}$ & CFI $^{\mathrm{b}}$ & TLI $^{\mathrm{b}}$ & RMSEA $^{\mathrm{c}}$ & ECVI $^{\mathrm{d}}$ \\
\hline M1 & 3,053 & 0,898 & 0,890 & 0,039 & 0,945 \\
M2 & 1,828 & 0,959 & 0,956 & 0,025 & 0,580 \\
M3 & 1,841 & 0,959 & 0,955 & 0,025 & 0,584 \\
M4 & 2,331 & 0,935 & 0,929 & 0,031 & 0,727 \\
\hline
\end{tabular}

Nota: ${ }^{a}>5$ - Mau. ]2;5] - Sofrível. ]1;2] - Bom. 1 - Muito bom. ${ }^{\text {b}}<0,8$ - Mau. [0,8;0,9[ - Sofrível. [0,9;0,95[- Bom. $\geq 0,95$ - Muito bom. ${ }^{c}>0,10$ -

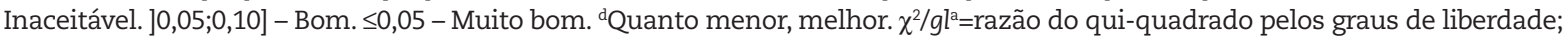
CFI=Comparative Fit Index; TLI=Tucker-Lewis Index; RMSEA=Root Mean Square Error of Aproximation; ECVI=Expected Cross-validation Index. 
O segundo componente extraído na ACP Orientação - reuniu os mesmos dez itens agrupados no estudo de Folstein et al. (1975). Embora sejam funções importantes a serem avaliadas no contexto da cognição, autores, como Carnero-Pardo (2013), argumentam que valerse de 10 itens para avaliar a orientação em um instrumento com um total de 30 itens pode ser exagero. O MEEM não foi criado apenas para rastrear declínio cognitivo em casos de demência, e a desorientação espacial, na maioria dos casos de demências, ocorre a partir de estágios intermediários. Assim, esses itens não discriminariam corretamente pessoas em fases iniciais de processos demenciais ou mesmo em casos de comprometimento cognitivo leve.

O terceiro componente foi formado por seis itens que exigem Memória de Curto Prazo e, por isso, assim foi denominado. Porém, alerta-se que um item ("dobra a folha") apresentou carga fatorial inaceitável, ou seja, abaixo de 0,30. A memória de curto prazo é uma função que deve necessariamente ser avaliada em um instrumento de rastreio de declínio cognitivo em pessoas idosas, visto que é um dos primeiros tipos de memória a ser comprometido com o envelhecimento (Yassuda, Viel, Silva, \& Albuquerque, 2011). Trata-se da capacidade de armazenar informações com retenção por um curto período de tempo e é dependente do processamento prévio pela memória de trabalho (Baddeley, 2011).

O quarto componente, Memória - Evocação, reuniu itens que exigem a recuperação de conteúdo exposto em momentos iniciais da testagem. A ACP realizada neste estudo identificou, por fim, um quinto componente, Memória - Reconhecimento, que agrupa itens que exigem a habilidade de reconhecer os objetos (caneta, relógio e folha de papel) e uma parte do próprio corpo (a mão direita), previamente armazenados na memória de longo prazo.

Apesar de ter a função única de lembrar informações, a memória contém vários subtipos que são afetados de maneiras distintas no envelhecimento (Yassuda, Viel, Silva, \& Albuquerque, 2011) e se manifestam conforme o processo de percepção, codificação, armazenamento e recuperação de conteúdos, sendo dividida, em princípio, em dois grandes grupos: curto prazo e longo prazo (Baddeley, 2011). A ACP do MEEM evidenciou essas divisões em processos e tipos ao constituir três componentes relacionados a processos mnemônicos. Porém, o exame conta com um número limitado de itens para avaliá-los (Carnero-Pardo, 2013). Eles devem ser devidamente rastreados em caso de suspeita de declínio cognitivo, visto que a perda ou diminuição do funcionamento da memória costuma ser uma das primeiras manifestações de prejuízo na cognição (APA, 2014).

Ainda que imbricações sejam perceptíveis, a ACP identificou e a AFC corroborou uma estrutura interna para o MEEM distinta das propostas por Folstein et al. (1975) e Castro-Costa et al. (2009). As convergências entre esses autores e os resultados desta investigação ocorrem principalmente na Orientação e na Memória de Curto Prazo. Entretanto, no caso de Castro-Costa et al. (2009; 2014), dois itens do componente Orientação apresentaram cargas inaceitáveis; um deles, "que período do dia é esse" — na versão do MEEM utilizada neste estudo, questiona-se: "que horas são agora?" - obteve carga fatorial muito boa no fator que denominaram Linguagem e Praxia.

Sobre a dimensão Memória de Curto Prazo, a estrutura fatorial não foi idêntica à proposta por Folstein et al. (1975) e nem à obtida por Castro-Costa et al. (2009). No primeiro caso, o trio de itens de recordação imediata foi chamado de registro e os demais itens foram agrupados numa dimensão chamada Linguagem. No segundo caso, a repetição imediata das três palavras foi agrupada em um componente chamado de atenção, a repetição da frase exibiu cargas fatoriais mais altas no fator denominado Linguagem e Praxia, e os dois itens de obediência ao comando de três etapas não apresentaram cargas fatoriais aceitáveis.

Outro fator desta ACP compreendido por itens que rastreiam o funcionamento da Memória - Evocação - apresentou uma estrutura parecida com as propostas de Folstein et al. (1975) e de Castro-Costa et al. (2009). Entretanto, os primeiros chamaram a dimensão proposta de Recordação e Castro-Costa et al. (2009) denominaram-na de Memória.

O arranjo de itens do componente Memória Reconhecimento foi expressivamente diferente dos modelos analisados. Em Folstein et al. (1975), esses itens fizeram parte da dimensão Linguagem, e em Castro-Costa et al. (2009) os itens de reconhecimento dos objetos "caneta" e "relógio" alcançaram cargas fatoriais inaceitáveis, entretanto discretamente mais altas no componente Memória. O item "pega a folha", apesar de ter também apresentado carga inexpressiva, pode ser alocado no componente Linguagem e Praxia.

Os itens do "sete seriados" mantêm-se juntos nos três modelos analisados. Contudo, tanto a denominação das dimensões em que foram incluídos quanto a presença de outros itens não foram as mesmas nos três modelos. Tanto o modelo de Folstein et al. (1975) quanto o de Castro-Costa et al. (2009) apresentam dimensões compostas exclusivamente por esses itens, ainda que as nomeiem de modo distinto: Atenção e Cálculo e Concentração, respectivamente. Essas terminologias podem ser consideradas inadequadas, já que esses itens demandam essencialmente subtração e atenção concentrada, uma exigência que é comum aos demais itens do MEEM. Destaca-se, entretanto, a força desse conjunto de itens, visto que, na ACP, foram aqueles com maiores cargas fatoriais.

A multidimensionalidade do MEEM também foi atestada pelas AFCs. Comparando os modelos de Folstein et al. (1975), Castro-Costa et al. (2009; 2014) e o modelo fatorial derivado da ACP, que foi obtido nesta pesquisa, observou-se que o último se mostrou mais ajustado. Ressalta-se que o modelo original também apresentou bons índices de ajuste. Do ponto de vista teórico, é possível afirmar que o modelo derivado da ACP é mais coerente com os atuais conhecimentos sobre processos psicológicos básicos e mais rigoroso em sua terminologia. Reitera-se que tanto Folstein et al. (1975) quanto Castro-Costa et al. (2009) empregaram designações questionáveis para as dimensões que adotaram.

Outro aspecto fundamental da psicometria precisa ser analisado: a padronização. Ela regula a uniformidade 
da medida, do processo de aplicação e de sua avaliação (Urbina, 2007), e a falta dela pode explicar, por exemplo, as diferenças entre os resultados de Castro-Costa et al. (2009) e os desta pesquisa, que utilizaram diferentes versões do MEEM. Variantes distintas desse instrumento são encontradas em língua portuguesa, apresentando traduções, números de itens e pontos de corte distintos, o que dificulta a comparação entre resultados de pesquisas (Melo \& Barbosa, 2015), especificamente as que produziram modelos fatoriais.

Com base nos resultados, é sensato afirmar que, além do total do MEEM, é possível usar dimensões desse exame, sejam elas as originalmente propostas por Folstein et al. (1975) ou as identificadas com a ACP e corroboradas com a AFC neste estudo. Considerar dimensões para o MEEM pode ser vantajoso porque isso permite o exame de condições clínicas específicas. Destaca-se que diferentes doenças cerebrais exibem diferentes subtipos de comprometimentos cognitivos, gerando manifestação de distintas combinações de sintomas (Castro-Costa et al., 2014), o que demanda avaliações particulares. Por exemplo, ao considerar especificamente a estrutura interna do
MEEM identificada com a ACP e corroborada pela AFC, nota-se que há dimensões mais ou menos sujeitas à influência da escolaridade, tendo sido uma delas inclusive designada Habilidades Escolares. Assim, ter escores para as diferentes partes da medida pode ser útil para analisar os efeitos da escolaridade no desempenho.

Em suma, este estudo analisou as evidências de validade baseadas na estrutura interna do MEEM (Brucki et al., 2003) e identificou indicadores de multidimensionalidade para ele. Todavia, no caso da ACP, a variância explicada pelos componentes pode ser considerada pequena, alguns deles possuem poucos itens, o valor próprio do primeiro fator é muito maior que o dos demais e todos os itens saturam positivamente no primeiro fator na matriz não rotacionada. A AFC revelou evidências de validade baseadas na estrutura interna para o MEEM. Apesar disso, recomenda-se retestar a estrutura interna do exame com amostras distintas (por exemplo, amostra clínica), e é recomendável proceder à análise da invariância fatorial além de investigar as propriedades psicométricas do MEEM por meio da Teoria de Resposta ao Item e não somente por meio de análises derivadas da Teoria Clássica dos Testes.

\section{Referências}

Abraham, I. L., Manning, C. A., Snustad, D. G., Brashear, H. R., Newman, M. C., \& Wofford, A. B. (1994). Cognitive screening of nursing home residents: factor structures of the Mini-Mental State Examination. Journal of the American Geriatrics Society, 42(7), 750-756. doi: 10.1111/j.1532-5415.1994.tb06536.x

American Educational Research Association, American Psychological Association, \& National Council on Measurement in Education [AERA, APA, \& NCME]. (2004). Standards for educational and psychological testing, Washington, DC: AERA.

American Psychiatry Association [APA]. (2014). Manual Diagnóstico e Estatístico de Transtornos Mentais (DSM-5) (5 ed.). Porto Alegre: Artmed.

Aprahamian, I., Martinelli, J. E., Cecato, J., \& Yassuda, M. S. (2011). Screening for Alzheimer's disease among illiterate elderly: accuracy analysis for multiple instruments. Journal of Alzheimer's Disease, 26(2), 221-229. doi: 10.3233/JAD-2011-110125

Baddeley, A. (2011). Memória de curta duração. Em A. Baddeley, M. C. Anderson \& M. W. Eysenck. (Orgs.), Memória (pp.31-53). Porto Alegre, RS: Artmed.

Baños, J. H., \& Franklin, L. M. (2002). Factor structure of the Mini-Mental State Examination in adult psychiatric inpatients. Psychological Assessment, 14(4), 397-400. doi: 10.1037/1040-3590.14.4.397

Beavers, A. S., Lounsbury, J. W., Richards, J. K., Huck, S. W., Skolits, G. J., \& Esquivel, S. L. (2013). Practical considerations for using exploratory factor analysis in educational research. Practical Assessment, Research \& Evaluation, 18(6), 1-13.

Bertolucci, P. H. F., Brucki, S. M. D., Campacci, S. R., \& Juliano, Y. (1994). O Mini exame do Estado Mental em uma população geral. Impacto da escolaridade. Arquivos de Neuropsiquiatria, 52(1), 1-7. doi: 10.1590/S0004-282X1994000100001

Beyermann, S., Trippe, R. H., Bähr, A. A., \& Püllen, R. (2013). Mini-Mental State Examination in geriatrics. An evaluation of diagnostic quality. Zeitschriftfiur Gerontologie und Geriatrie, 46(8), 740-747. doi: 10.1007/s00391-013-0488-6

Braekhus, A., Laake, K., \& Engedal, K. (1992). The Mini-Mental State Examination: identifying the most efficient variables for detecting cognitive impairment in the elderly. Journal of the American Geriatrics Society, 40(11), 1139-1145. doi: 10.1111/j.1532-5415.1992.tb01804.x

Brucki, S. M. D., Mansur, L. L., Carthery-Goulart, M. T., \& Nitrini, R. (2011). Formal education, health literacy and Mini-Mental State Examination. Dementia and Neuropsychologia, 5(1), 26-30.

Brucki, S. M. D, Nitrini, R., Caramelli, P., Bertolucci, P. H. F., \& Okamoto, I. H. (2003). Suggestions for utilization of the mini-mental state examination in Brazil. Arquivos de Neuropsiquiatria, 61(3B), 777-781. doi: 10.1590/S0004-282X2003000500014

Brugnolo, A., Nobili, F., Barbieri, M. P., Dessi, B., Ferro, A., Girtler, N., Palummeri, E., Partinico, D., Raiteri, U., Regesta, G., Servetto, G., Tanganelli, P., Uva, V., Mazzei, D., Donadio, S., De Carli, F., Colazzo, G., Serrati, C., \& Rodriguez, G. (2009). The factorial structure of the Mini Mental State Examination (MMSE) in Alzheimer's disease. Archives of Gerontology and Geriatrics, 49(1), 180-185. doi: 10.1016/j.archger.2008.07.005

Carnero-Pardo, C. (2013). ¿Es hora de jubilar al Mini-Mental?. Neurologia, 29(8), 473-481.

Castro-Costa, É., Dewey, M. E., Uchôa, E., Firmo, J. O., Lima-Costa, M. F., \& Stewart, R. (2014). Construct validity of the mini mental state examination across time in a sample with low-education levels: 10-year follow-up of the Bambuí Cohort Study of Ageing. International Journal of Geriatric Psychiatry, 29(12), 1294-1303. doi: 10.1002/gps.4113

Castro-Costa, E., Fuzikawa, C., Ferri, C., Uchoa, E., Firmo, J., Lima-Costa, M. F., Dewey, M. E., \& Stewart, R. (2009). Dimensions underlying the Mini-Mental State Examination in a sample with low-education levels: the Bambui Health and Aging Study. The American Journal of Geriatric Psychiatry, 17(10), 863-872. doi: 10.1097/JGP.0b013e3181ab8b4d 
Commenges, D., Gagnon, M., Letenneur, L., Dartigues, J. F., Barberger-Gateau, P., \& Salamon, R. (1992). Statistical Description of the Mini-Mental State Examination for French Elderly Community Residents. The Journal of Nervous and Mental Disease, 180(1), $28-32$.

Damásio, B. F. (2012). Uso da análise fatorial exploratória em psicologia. Avaliação Psicológica, 11(2), 213-228.

Farfel, J. M., Nitrini, R., Suemoto, C. K., Grinberg, L. T., Ferretti, R. E. L., Leite, R. E. P., ... \& Rodriguez, R. D. (2013). Very low levels of education and cognitive reserve. A clinic pathologic study. Neurology, 81(7), 650-657. doi: 10.1212/WNL.0b013e3182a08f1b

Fillenbaum, G. G., Heyman, A., Wilkinson, W. E., \& Haynes, C. S. (1987). Comparison of two screening tests in Alzheimer's disease: The correlation and reliability of the Mini-Mental State Examination and the Modified Blessed Test. Archives of Neurology, 44(9), 924-927. doi: 10.1001/archneur.1987.00520210026014

Folstein, M. F., Folstein, S. E., \& Mchugh, P. R. (1975). Mini Mental State. A practical method for rading the cognitive state of patients for the clinician. Journal of Psychiatric Research, 12(3), 189-198. doi: 10.1016/0022-3956(75)90026-6

Folstein, M. F., Folstein, S. E., \& McHugh, P. R. (2007). Reply. Acta Psychiatrica Scandinavica, 116(2), 157. doi: 10.1111/j.1600-0447.2007.01038.x

Hoops, S., Nazem, S., Siderowf, A. D., Duda, J. E., Xie, S. X., Stern, M. B., \& Weintraub, D. (2009). Validity of the MoCA and MMSE in the detection of MCI and dementia in Parkinson disease. Neurology, 73(21), 1738-1745. doi: 10.1212/WNL.0b013e3181c34b47

Ideno, Y., Takayama, M., Hayashi, K., Takagi, H., \& Sugai, Y. (2012). Evaluation of a Japanese version of the Mini-Mental State Examination in elderly persons. Geriatrics \& Gerontology International, 12(2), 310-316. doi: 10.1111/j.1447-0594.2011.00772.x

Jones, R. N., \& Gallo, J. J. (2000). Dimensions of the Mini-Mental State Examination among community dwelling older adults. Psychological Medicine, 30(03), 605-618. doi: 10.1017/S0033291799001853

Kim, J. W., Lee, D. Y., Seo, E. H., Sohn, B. K., Choe, Y. M., Kim, S. G., ... \& Kim, K. W. (2014). Improvement of screening accuracy of Mini-Mental State Examination for mild cognitive impairment and non-Alzheimer's disease dementia by supplementation of verbal fluency performance. Psychiatry Investigation, 11(1), 44-51. doi: 10.4306/pi.2014.11.1.44

Laros, J. A. (2012). O uso da análise fatorial: algumas diretrizes para pesquisadores. Em L. Pasquali (Ed.) Análise fatorial para pesquisadores (pp. 141-160). Brasília: LabPAM Saber e Tecnologia.

Markus, K. A., \& Borsboom, D. (2011). Reflective measurement models, behavior domains, and common causes. New Ideas in Psychology, 31(1), 54-64. doi: 10.1016/j.newideapsych.2011.02.008

Marôco, J. (2010). Análise de equações estruturais: fundamentos teóricos, software e aplicações. Lisboa: Report Number.

Melo, D. M., \& Barbosa, A. J. G. (2015). O uso do Mini-Exame do Estado Mental em pesquisas com idosos no Brasil: uma revisão sistemática. Revista Ciência E Saúde Coletiva, 20(12), 3865-3876. doi: 10.1590/1413-812320152012.06032015

Mitolo, M., Salmon, D. P., Gardini, S., Galasko, D., Grossi, E., \& Caffarra, P. (2014). The new Qualitative Scoring MMSE Pentagon Test (QSPT) as a valid screening tool between autopsy-confirmed dementia with Lewy bodies and Alzheimer's disease. Journal of Alzheimer's Disease, 39(4), 823-832. doi: 10.3233/JAD-131403

Molloy, D. W., \&Standish, T. I. (1997). Mental status and neuropsychological assessment: a guide to the standardized Mini-Mental State Examination. International Psychogeriatrics, 9(S1), 87-94. Recuperado de http://www.dementia-assessment.com.au/guidelines/guide_standardised_mmse.pdf

Muthén, L K, \& Muthén, B. O. (2012). Mplus: Statistical analysis with latent variables. User's guide. Los Angeles: Muthén \& Muthén.

Neri, A. L., Yassuda, M. S., Araújo, L. F. D., Eulálio, M. D. C., Cabral, B. E., Siqueira, M. E. C. D., ... \& Moura, J. G. D. A. (2013). Metodologia e perfil sociodemográfico, cognitivo e de fragilidade de idosos comunitários de sete cidades brasileiras: Estudo FIBRA. Caderno de Saúde Pública, 29(4), 778-792. doi: 10.1590/S0102-311X2013000400015

Nitrini, R., Caramelli, P., Bottino, C. M. C., Damasceno, B. P., Brucki, S. M. D., \& Anghinah, R. (2005). Diagnóstico de doença de Alzheimer no Brasil: avaliação cognitiva e funcional. Recomendações do Departamento Científico de Neurologia Cognitiva e do Envelhecimento da Academia Brasileira de Neurologia. Arquivos de Neuropsiquiatria, 63A(3), 720-27. doi: 10.1590/S0004-282X2005000400033

Schultz-Larsen, K., Kreiner, S., \& Lomholt, R. K., 2007. Mini-Mental Status Examination: mixed Rasch model item analysis derived two different cognitive dimensions of the MMSE. Journal of Clinical Epidemiology, 60(3), 268-279. doi: 10.1016/j.jclinepi.2006.06.007

Seabra, M. L. V., Concilio, G., Villares, J. B., \& Carlini, E. A. (1990). Avaliação do teste "Mini-Mental State" em voluntários e pacientes brasileiros. Revista $A B P$-APL, 12, 1-29.

Spencer, R. J., Wendell, C. R., Giggey, P. P., Katzel, L. I., Lefkowitz, D. M., Siegel, E. L., \& Waldstein, S. R. Psychometric limitations of the Mini-Mental State Examination among nondemented older adults: An evaluation of neurocognitive and magnetic resonance imaging correlates. Experimental Aging Research, 39(4), 382-397. doi: 10.1080/0361073X.2013.808109.

Tinklenberg, J., Brooks, J. O., Tanke, E. D., Khalid, K., Poulsen, S. L., Kraemer, H. C., ... \& Yesavage, J. A. (1990). Factor analysis and preliminary validation of the mini-mental state examination from a longitudinal perspective. International Psychogeriatrics, 2(2), 123-134. doi: 10.1017/ S1041610290000382

Urbina, S. (2007). Fundamentos da testagem psicológica. Porto Alegre: Artmed.

Vasconcelos, L. G., Brucki, S. M. D., \& Bueno, O. F. A. (2007). Cognitive and functional dementia assessment tools: Review of brazilian literature. Dementia \& Neuropsychologia, 1(1), 18-23. doi: 10.1590/S1980-57642008DN10100004

Yassuda, M. S., Viel, T. A., Silva, T. B. L., \& Albuquerque, M. S. (2011). Memória e envelhecimento: aspectos cognitivos e biológicos (pp. 1477-1483). Em E. V. Freitas \& L. Py (Eds.). Tratado de Geriatria e Gerontologia (3 $3^{\text {a }}$ ed.). Rio de Janeiro: Guanabara Koogan.

recebido em março de 2016 reformulado em outubro de 2016 aprovado em janeiro de 2017

\section{Sobre os autores}

Denise Mendonça de Melo é Psicóloga, doutora em Psicologia pela Universidade Federal de Juiz de Fora, mestre em Gerontologia pela Unicamp e Docente do Centro Universitário Estácio Juiz de Fora. Recebeu apoio financeiro da Capes para a realização da pesquisa.

Altemir José Gonçalves Barbosa é Psicólogo, Professor Titular da Faculdade de Psicologia e Docente do Programa de Pós-Graduação em Psicologia da Universidade Federal de Juiz de Fora.

Anita Liberalesso Neri é Psicóloga, Professora Titular da Faculdade de Educação e Docente do Programa de Pós-Graduação em Gerontologia da Faculdade de Ciências Médicas da Unicamp. 